\title{
ACCURATELY MEASURING HEALTH OVER THE LIFE COURSE
}

Fabian Lange and Doug McKee

CRR WP 2010-5

Released: May 2010

Date Submitted: April 2010

Center for Retirement Research at Boston College

Hovey House

140 Commonwealth Avenue

Chestnut Hill, MA 02467

Tel: 617-552-1762 Fax: 617-552-0191

The research reported herein was pursuant to a grant from the U.S. Social Security Administration (SSA) funded as part of the Retirement Research Consortium (RRC). The findings and conclusions expressed are solely those of the authors and do not represent the views of SSA, any agency of the Federal Government, the RRC, Yale University, or Boston College.

(C) 2010, by Fabian Lange and Doug McKee. All rights reserved. Short sections of text, not to exceed two paragraphs, may be quoted without explicit permission provided that full credit, including (C) notice, is given to the source. 


\title{
About the Sandell Grant Program
}

This paper received funding from the Steven H. Sandell Grant Program for Junior Scholars in Retirement Research. Established in 1999, the Sandell program's purpose is to promote research on retirement issues by scholars in a wide variety of disciplines, including actuarial science, demography, economics, finance, gerontology, political science, psychology, public administration, public policy, sociology, social work, and statistics. The program is funded through a grant from the Social Security Administration (SSA). For more information on the Sandell program, please visit our web site at $h t t p: / / c r r . b c . e d u / o p p o r t u n i t i e s / s t e v e n \_h . \_s a n d e l l \_$ grant_program_2.html, send e-mail to crr@bc.edu, or call Marina Tsiknis at (617) 552-1092.

\section{About the Center for Retirement Research}

The Center for Retirement Research at Boston College, part of a consortium that includes parallel centers at the University of Michigan and the National Bureau of Economic Research, was established in 1998 through a grant from the Social Security Administration. The Center's mission is to produce first-class research and forge a strong link between the academic community and decision makers in the public and private sectors around an issue of critical importance to the nation's future. To achieve this mission, the Center sponsors a wide variety of research projects, transmits new findings to a broad audience, trains new scholars, and broadens access to valuable data sources.

\section{Center for Retirement Research at Boston College}

\author{
Hovey House \\ 140 Commonwealth Avenue \\ Chestnut Hill, MA 02467 \\ phone: 617-552-1762 fax: 617-552-0191 \\ e-mail: crr@bc.edu \\ crr.bc.edu
}

Affiliated Institutions:

The Brookings Institution

Massachusetts Institute of Technology

Syracuse University

Urban Institute 


\begin{abstract}
This paper develops a new method of estimating rich, dynamic models of health based on multiple health measures available in the HRS. We apply these methods to investigate what generates the large socioeconomic gradient in health. Preliminary results suggest a large role for initial differences in health at age 50 that persist into old age.
\end{abstract}




\title{
The Role of Dynamics in Health-Education Gradient
}

\author{
Fabian Lange and Doug McKee (Yale University)
}

April 9th, 2010

\section{$1 \quad$ Introduction $^{1}$}

Countless studies find that health and socioeconomic status are closely linked.

On the face of it, how health and SES co-vary is so well documented that there seems little need to again take up the question how health varies with SES and how this variation changes with age. Whether health is measured by survival, by subjective measures such as self-reported health status (SRHS), by objective health measures such as grip strength, or by virtually any other measure, the same picture emerges: high socioeconomic status (SES) cor-

\footnotetext{
${ }^{1}$ The research reported herein was pursuant to a Sandell grant from the U.S. Social Security Administration (SSA) funded as part of the Retirement Research Consortium (RRC). The findings and conclusions expressed are solely those of the authors and do not represent the views of SSA, any agency of the Federal Government, the RRC, Yale University, or Boston College.
} 
relates with better health. ${ }^{2}$ Figure 1 illustrates this fact using 4 different measures from the Health and Retirement Study (HRS). Regardless of how one measures health, health is positively associated with SES.

However, closer inspection reveals that the strength of this association varies with the measure used. Even within SES, statements about how health

\footnotetext{
${ }^{2}$ The literature on health inequalities across education levels is vast. We reference here some recent papers. For hypertension and cholesterol see Cutler, Lange, Meara, Richards, and Ruhm (2009). For self-reported health, Halliday (2009). For mortality: Cutler et al. (2009) and Lleras-Muney (2005). For disability: Schenie, Freedman, and Martin (2009). For diabetes: Smith (2007). For research focusing specifically on health dynamics, consider Adams, et al. (2003), Meet, Miller, \& Rosen (2003), and Hurd and Kapteyn (2003), Halliday (2008, 2009), and Heiss, Börsch-Supan, Hurd, and Wise (2009).
} 
changes with age depend on the measure. Using SRHS or the Large Muscle Index, we might be tempted to conclude that 50-year-old drop-outs are on average of the same health as 80-year-old high school graduates. But, using expiratory air flow, we might instead conclude that the health of 50-year-old drop-outs is on par with that of 65-year-old high school graduates. And, using log grip strength to measure health, we might again change our mind and conclude that the health of 50-year-old drop-outs is equivalent to that of 55-year-old graduates.

Much of what we think we know about the Health-SES gradient is therefore measure-dependent and which measure one uses will influence the answers given to questions such as how the SES gradient varies with age. Further, what measure of health one uses will affect whether differences in health endowments, in the incidence of severe disease episodes, or in the rates of functional decline are found to generate the SES gradient. And, as long as different researchers do not agree on the answers to these questions solely because they use different health measures, there will also be no way to agree on answers to questions such as what drives health inequality in the US or what policies should be implemented to address the widening gap in health.

There is thus a need for describing the health process in a parsimonious manner that combines the information contained in many different health measures and is independent of the particular measure used to measure health. The representation of health needs to be parsimonious to allow sys- 
tematic investigation of the health process in a tractable manner. ${ }^{3}$ At the same time, the representation of health needs to be general in the sense that it is invariant to the health measure used. Without such invariance across health measures, consensus and progress in understanding health dynamics will be elusive. In this paper, we propose a method of estimating dynamic models of health that both uses the information available in multiple health measures and allows description of the evolution of health in a parsimonious manner.

Our method adapts and combines two techniques first developed in different contexts. In a first step, we use factor analytic methods to estimate how health measures are related to an underlying latent variable called "health." 4 For each age, we can identify how this latent variable is distributed by examining how the various health measures in the data are correlated. ${ }^{5}$ We can also identify the joint distribution of the vector of health measures and the latent health variable. ${ }^{6}$

We then model how the latent health variable evolves stochastically over time. We estimate the parameters of this dynamic model using the method

\footnotetext{
${ }^{3}$ The focus on analyzing health measures in isolation has been driven by the desire for parsimonious representations of health as well as the lack of rich health data in some commonly used data-sets such as the PSID. The increasing availability of rich data on health in large panel data sets such as the Health and Retirement Study (HRS) makes it increasingly hard to justify analzing individual health measures in isolation. The need to describe the dynamics of health in a parsimonious manner however remains.

${ }^{4}$ Factor models are a common data reduction strategy familiar in the psychometric literature. In health, related methods are used by Halliday (2008, 2009), Heiss, Hurd, and Wise (2009), and Meijer, Kaptyn, and Andreyeva (2008).

${ }^{5}$ This distribution is non-parametrically identified.

${ }^{6}$ This joint distribution is only parameterically identified.
} 
of simulated moments (McFadden, 1989). ${ }^{7}$ We minimize the distance between moments of the implied and the observed joint distribution of health measures across age. To derive the moments implied by our model, we simulate the latent health process and use the static measurement model that we estimated in the first step to map the simulated health process into a sequence of health measures.

In addition to summarizing the information from multiple health measures in a parsimonious way, our method has a number of advantages. First, because we separately estimate the static measurement and the dynamic health model, the estimated age-conditional distribution of health will be robust to misspecifying the dynamic health model. Second, because we have multiple health measures and because our health measures include continuous measures, we can non-parametrically identify ${ }^{8}$ the distribution of the underlying health variable. This ability to consider distributions that deviate from normality turns out to be empirically important. Finally, and most importantly, our method allows us to specify and estimate dynamic models of health that are substantially richer than existing models.

We implement our method using data from the HRS. For up to 16 years of follow-up, the HRS panel provides rich data on the health of older individuals. We rely on 3 continuous objective measures of health (grip strength, expiratory air flow, and walking speed) as well as two subjective measures

\footnotetext{
${ }^{7}$ Simulation methods are becoming increasing popular in structural labor economics and applied macroeconomics.

${ }^{8}$ In practice, we estimate flexible, but still parametric distributions of health.
} 
of health (a "large muscle index" and SRHS). These masures allow us to estimate very flexible distributions of latent health at different ages. We find that the distribution of latent health is very far from normal, it is often bimodal and skewed to the left. These findings are generally not reproduced by current dynamic models that are estimated in the literature.

We are still developing our dynamic model. In this paper, we assume that the latent health variable follows a single lag autoregressive (AR-1) process with time-varying parameters and we allow for endogenous mortality by relating the probability of death to the underlying health variable and age.

Estimates of this simple dynamic model generate a number of interesting results. First, we find that endogenous mortality can not account for the narrowing in the SES gradient observed at ages $75+$. Second, we find substantial individual persistence in the health dynamics and, in fact, the evolution of health looks very similar to a random walk. Third, across education groups we find quite similar parameter estimates for the dynamic specification and also for the mortality model. Together, we are tentatively willing to hypothesize that the observed SES health gradient is not driven by differences in the dynamic process governing health dynamics after age 50. Instead, both education groups experience similar health processes, but these processes have sufficient persistence that existing differences in the SES gradient at age 50 persist until relatively old age. At this point, however, we want to emphasize that this result is very preliminary and that more analysis 
and estimates from richer dynamic models are needed to become confident about these findings.

We proceed as follows. In Section 2, we describe the dynamic model of health and the static health measurement model. In Section 3, we describe the data that forms the basis of our estimating health measures. We show, using the health measures contained in the HRS, that the observed variation in health with age and education depends significantly on the health measure used. Section 4 presents results from estimating the static measurement model and Section 5 presents the method we use to estimate the dynamic model and presents the corresponding estimation results. Section 6 concludes.

\section{A Model of Health and Health Measure- ment}

We begin by describing our assumptions about the data generating process (DGP) behind the evolution and measurement of health. The model can be separated into a dynamic model of latent health and a measurement model that describes how latent health maps into observed health measures. In later Sections, we estimate this model on the population of males for two different education rates. In our exposition of the model, we will keep the dependence on education implicit. To simplify the notation, we will also suppress individual subscripts. 


\subsection{The Dynamics of Health}

We assume that health of an individual at age a can be represented by a scalar $h_{a}$. For now, we assume that $h_{a}$ follows a first order autoregressive process with drift:

$$
h_{a+1}=\mu_{g}+\rho_{g} h_{a}+v_{a}
$$

The parameters that describe this process are the constant $\mu_{g}$, the autoregressive parameter $\rho_{g}$ and the variation of the innovation $\sigma_{v, g}^{2}$. The innovation $v_{a}$ captures idiosyncratic shocks to individuals' health and is normally distributed. The parameters are indexed by g, which denotes the 5-year age group an individual belongs to. We therefore allow the dynamic process governing health to change with age. ${ }^{9}$

We initialize the random process (1) by specifying an initial distribution $F\left(h_{0}\right)$ that describes health. This initial distribution is based on an estimate of the distribution of health for the first age group observed in our data that we obtain using the static measurement model described in Section 5.2 below.

The second equation of our dynamic model describes mortality. We assume that individual mortality depends on the health status in the following manner:

$$
\operatorname{Pr}\left(s_{a}=1\right)=\Phi\left(\alpha_{0, g}+\alpha_{1, g} h_{a}\right)
$$

where $s_{a}$ is an indicator for whether an individual survived to age a +1 condi-

\footnotetext{
${ }^{9}$ In principle, we could allow the parameters to vary for each age of the individuals. However, limited sample size forces us to group individuals into 5-year age groups.
} 
tional on being alive at age a, and $\Phi($.$) denotes the cumulative distribution$ function of the standard normal. Again, we allow the parameters to vary by age group.

\subsection{The Measurement of Health}

Rather than observing $h_{a}$ directly, we observe categorical and continuous measures of health. Let $Y_{a}$ stand for a $m_{1}$ vector of categorical indicator variables and $X_{a}$ for a $m_{2}$-vector of continuous indicator variables $x_{m}$. The total number of manifest categorical and continuous measurements is $m=$ $m_{1}+m_{2}$. Each categorical variable $y_{j, a} \in Y$ is assumed to reflect an underlying

latent index $\widetilde{y}_{j, a}$ and cutoffs $c_{j, g}^{k}$. We also assume that each categorical variable is ordered and has $K j$ segments.

Thus, each $y_{j} \in Y$ is linked to its latent counterpart $\widetilde{y_{j}} \in \tilde{Y}$ :

$$
y_{j, a}=\Sigma_{k=1}^{K_{j}} 1\left(\widetilde{y}_{j, a} \geq c_{j, g}^{k-1}\right)
$$

where $1($.$) is an indicator function taking the value 1$ if the condition in parenthesis is true and 0 otherwise.

Collect the latent indices $\widetilde{y}_{j, a}$ and the continuous measurement variables $x_{j, a}$ in a vector $Z_{a}$. The measurements $Z_{a}$ and $h_{a}$ are linearly related with error $\epsilon_{a}$ :

$$
Z_{a}=\left(\begin{array}{c}
X_{a} \\
\widetilde{Y}_{a}
\end{array}\right)=\alpha_{g}+\Lambda_{g}^{\prime} h_{a}+\epsilon_{a}
$$

Here, $\alpha_{g}$ denotes an m-vector of intercepts, $\Lambda_{g}$ denotes an m-vector of 
factor loadings and $\epsilon_{a}$ an m-vector of independently distributed measurement errors. ${ }^{10}$

The equations (3) and (4) define the measurement model. The parameters in this measurement model are all subscripted by g, reflecting the fact that we estimate a different measurement model for each of the 5-year age groups.

At this point, it becomes necessary to comment briefly on the identification of the measurement model. ${ }^{11}$ We need to identify the parameters $\left(\alpha_{g}, \Lambda_{g}\right)$, the vector of the variances of $\epsilon_{a}$, and the distribution $\mathrm{F}_{g}($.$) of the$ latent health variable $h_{a}$. Standard factor analytic results imply that, assuming that we have 3 or more measurement variables, we can identify these parameters only up to 2 normalizations, one for the scale and one for the location of the distribution of the latent health variable. We will impose these normalizations on the intercepts and the factor loading of one continuous variable. This implies that health is measured with respect to units of this first continuous measurement. We will always impose these normalizations on the same measurement, regardless of age and education. To the extent that this variable is objectively measured, we can use it to compare the level of health across ages and education.

We also need to impose a second set of normalizations to account for the fact that the index functions of ordinal categorical variables are only observed

\footnotetext{
${ }^{10}$ The assumption of independence is stronger than the uncorrelatedness assumption typical in factor analysis. It is required to obtain identification of $\mathrm{F}(\mathrm{h})$ without parameteric assumptions.

${ }^{11}$ We provide a more complete identification argument in Appendix 1.
} 
up to scale and location. We therefore normalize the intercepts and the error variances of the measurement equations dealing with categorical variables to 0 and 1 respectively.

As we show in the appendix, we can go beyond identifying the variance of $h_{a}$ and can non-parametrically identify the entire distribution $F_{g}\left(h_{a}\right)$. This is possible because we have access to several continuous measurement variables.

It is important to understand that we rely on only a cross-section of health measures at age a to identify the 'static' parameters $\left(\alpha_{g}, \Lambda_{g}, \operatorname{Var}_{g}\left(\epsilon_{a}\right)\right)$ and the distribution $F_{g}\left(h_{a}\right)$. By contrast, we need longitudinal data on health

measures across age to identify the dynamic parameters $\left(\mu_{g}, \rho_{g}, \sigma_{g}^{2}, \beta_{0, g}, \beta_{1, g}\right)$.

\section{Data Description}

For our study, we need a large survey with socioeconomic data and rich data on individual health. We require continuous measurements of health, but we can also utilize ordinal categorical health measures. The data can contain both subjective and objective measures. Ideally, the data will follow individuals over as long a period as possible. As a nationally representative longitudinal study, the HRS is ideally suited for our purposes. Since 1992, the HRS regularly collects data with nearly 20,000 respondents representing the US population aged 50 and older. In order to maintain a representative sample of the population aged $50+$, new birth cohorts are enrolled every 6 years. We base our study on the 10 surveys conducted between 1992 and 
$2006 .^{12}$

Our data is based on the RAND HRS data files, the HRS Tracker files and the physical measure files for the 2004 and 2006 waves. The RAND files (version $\mathrm{H}$ ) are a user friendly version of the HRS made available by the RAND corporation and we obtain the subjective health variables as well as the education and age variables from the RAND files. In addition, we merge in 3 objective health measures (peak expiratory air flow, hand grip strength and timed walking speed data) from the 2004 and 2006 physical measure files. The HRS Tracker files (version 2.0, January 2008) provide Vital Stats based on the National Death Index. ${ }^{13}$

The HRS data contains a multitude of subjective health measures and we choose two from amongst these, an index of large muscle strength and the SRHS measure which reports subjective health status on a 5 point scale from excellent to poor. The index of large muscle strength is derived from variables indicating difficulties in four tasks: sitting for two hours, getting up from a chair, stooping, kneeling or crouching, and pushing or pulling a large object. ${ }^{14}$ In addition to the subjective health measures, we use 3 objective health measures which are available in the 2004 and 2006 waves.

\footnotetext{
${ }^{12}$ We use data from the 1992, 1993, 1994, 1995, 1996, 1998, 2000, 2002, 2004, and 2006 surveys. Recently data from the 2008 survey has become available and we plan to expand the scope of our analysis to cover this wave.

${ }^{13}$ The mortality data in the tracker files is based on finder files submitted to NCHS in 1995, 2000, 2002, and 2004. Based on the information in the tracker files, we can determine the vital status and the year and month of death up to 2004.

${ }^{14}$ The SHLT is available for all waves in the study, whereas the large muscle index is not available for waves 1 and for a subset of the sample in wave 2 .
} 
The hand grip strength measure is a measure of general muscle strength and of the presence of arthritis and other conditions in the hand. The measure of lung function, peak expiratory air flow, is a measure of obstructive lung disease. Declines in peak expiratory air flow have been shown to be related to mortality, cognitive decline and physical decline. Hand grip strength has also been shown to be related to general physical and medical status and predicts mortality. Finally, the timed walk, which has only been collected from individuals aged $65+$, has been shown to be a highly reliable measure of functional capacity and it predicts major health outcomes. There are a number of variables that we have not utilized, both subjective and objective. Our methodology allows use of these additional measures and indeed benefits from on having a large set of health measures. We therefore plan to extend our analysis to use a more complete set of health measures. ${ }^{15}$

Table 1 presents summary statistics for our sample, treating each individualyear observation as an independent observation. ${ }^{16}$ The age of the population runs from 50 to 109, with a mean of about 67 years. We have access to

\footnotetext{
${ }^{15} \mathrm{As}$ economists we are interested in the constraints that health limitations place on individuals' ability to participate in the labor force and in general on economic and social activities. We therefore would like health measures that are closely related to general functional status, rather than purely predictive of future health events. At this stage, we hope that conversations with epidemiologists and medical professionals will enable us to choose the appropriate health measures from among the set of variables available in the HRS.

${ }^{16}$ To facilitate the empirical analysis, it will be useful to put the variables on about the same scale. We will therefore standardize all continous variables using the entire population of the same gender, but across all ages and education levels. These variables will therefore have a mean of 0 and a standard deviation of 1 . We also find it useful to have all variables increase in health and therefore re-code the walking speed and the large muscle index, so that healthier individuals have higher values.
} 
the continuous objective measures of health only for 2004 and 2006. Comparing 2004 and 2006 with the earlier years, we find that the population is a little more than a year older, breaks down about equally into males and females, and is about equally educated (two-thirds of the population have a high school degree). Respondents report on average to be of good health and the average number of difficulties reported for tasks making up the large muscle index is about 1.3 (out of four). The large muscle index and SRHS indicate that the population is slightly less healthy during the years 2004 and 2006, but this is likely due to the fact that the population is somewhat older during the later years.

In this study, we examine how the educated and the less educated differ in their aging process. We will now take a first look at this question using the 
raw data. Figure 1, already discussed in the introduction, examines how the average health measures vary with age. The top panels show the large muscle index ${ }^{17}$ and a dichotomized version of the SRHS measure. ${ }^{18}$ The bottom two panels show the average log grip strength and the expiratory air flow measure. All of these measures display a significant SES gradient in health, but the gradient is significantly larger when one examines subjective measures (such as SRHS and the large muscle index) as opposed to objective measures. There are other important differences between the health measures. For instance, the objective measures decline almost linearly with age while health as measured by the self rated health variable and the mean large muscle index seems to decline at an increasing rate (prior to very old age). Depending on the health measure used, researchers will also come to different conclusions about the relative gradients in education and age. The health gradient in education relative to the age gradient is much larger if one relies on SRHS or the large muscle index than if one uses the measures of log grip strength, expiratory air flow, or log walking speed. All these findings suggest that estimates of the dynamic health process within and across education will be sensitive to the health measure used.

We thus find large qualitative differences in the aging process depending on the health measure used. This suggests the need to consider a broader

\footnotetext{
${ }^{17}$ We have inverted the large muscle index for this graph so that all indexes increase with better health.

${ }^{18}$ Responses of poor and fair health are classified as "bad health" and the remaining (good, very good, and excellent) are classified as "good health".
} 
set of measures of health than just the dichotomous measures of good health typically considered in the literature. We now explore this issue further. For this purpose, we consider how much information there is in the other health measures that is orthogonal to the good health measure, age, and gender. For this purpose, we regress the various health measures on a full set of pairwise interactions between age, gender, and good health ${ }^{19}$ - where age is measured using a vector of dummy variables, one for each age. We find that the Rsquares for this specification are $0.18,0.23,0.29$ and 0.14 for the large muscle index, expiratory air flow only, log grip strength, and log walking speed measures respectively. These are modest R-squares, indicating that age and the good health variable can explain some, but by no means all of the variation in the other health variables. The question then arises, whether the remaining variation in these health measures reflects purely noise or whether it in fact contains information about the health of individuals. Table 2 investigates this question by showing the correlations in the residuals from the above described regressions. ${ }^{20}$ If the residualized variation represented pure noise then we would expect these correlations to equal zero. Our interpretation of these large and statistically significant correlations is that there is indeed a lot of information in the health measures that is orthogonal to the self rated

\footnotetext{
${ }^{19}$ The variable "good health" is the dichotomous variable constructed from the selfreported health status variable and presented in figure 1 . This measure is commonly used in economic applications to summarize individual health.

${ }^{20}$ Remember that the large muscle index is inversely related with health, so that a larger index implies worse health. All other variables are positively related to health.
} 
health measure.

Overall, the above reported statistics strongly suggest that the commonly used dichotomous scalar health indices based on SRHS (eg. Halliday (2008, 2009) Heiss et al. (2008)) ignore a lot of the information about health contained in other health measures. The main advantage of such dichotomous scalar measures is that it is possible to describe how they evolve dynamically in a parsimonious manner. It is typically much harder to describe the evolution of a vector of health measures. ${ }^{21}$ We recognize the need to describe

\footnotetext{
${ }^{21}$ It is even harder to describe the evolution of a vector of health measures if they contain both continuous and categorical variables that can take on many values.
} 
the data in a parsimonious way, but we also assert that any study of the dynamics of health can only succeed if the measures on which it is based are comprehensive measures of health, a requirement that is not satisfied by simple indicators of "good" and "bad" health as are still commonly used in much of the literature.

Factor analytic methods allow us to reconcile these two conflicting objectives: they are based on information from many health measures and they summarize this information in low-dimensional vectors of latent health.

\section{Estimating the Distribution of Health - the Static Measurement Model}

We will now estimate how latent health is distributed within each age and education group. We estimate the distribution of health treating all observations from the same age in the HRS as a separate cross-section. Only in the next section, where we estimate the dynamic model, will we exploit the panel nature of the data. We thus pool all observations of individuals from the same age, regardless in what year these individuals appear in the HRS. Furthermore, we combine ages into 8 -year age bins, ranging from 50-54 to $85+$. For each of the age groups, we estimate the same specification, which we describe next.

We rely on the health measurements described in the previous section. We normalize the distributions of the continuous variables such that the 
population mean of the log grip, air flow, and walking speed variables are 0 and the standard deviation is 1 . We also impose the necessary normalizations on the intercept and factor loadings of the log grip strength variable. Thus, the latent health variable is measured in terms of (population) standard deviations of the log grip strength variable.

We assume that the latent health variable is distributed as a mixture of normal random variables:

$$
F_{g}\left(h_{a}\right)=p_{g, a} * N\left(\mu_{1, g}, \sigma_{1, g}^{2}\right)+\left(1-p_{g}\right) N\left(\mu_{2, g}, \sigma_{2, g}^{2}\right)
$$

Mixtures of normals deliver very general distributions and can accommodate the skewness, thick tails and bimodalities we observe in the data. We show these estimated distributions below.

Table 3 reports the point estimates of the model for those aged 50-54, 65-69, and 80-84 for both types of education. ${ }^{22}$

\footnotetext{
${ }^{22}$ To keep the table manageable, we only show the point estimates. Again, complete results are available from the authors upon request.
} 
As demonstrated in Table 4, we fit the data well. In that table we show moments of the joint distribution of the health measurements for the agegroup of 50-54 year old males with high school degrees. Clearly, the model does a very good job at fitting the joint distribution of the large muscle index and the self-reported health variable. Because we only have the log grip strength variable and the expiratory air flow variable for the years 2004 and 2006, the number of observations for which these variables are available is much smaller. This implies that the parameter estimates of the static model will primarily be driven by the subjective health measures. Nevertheless, we also capture some of the joint variation in the objective health measures, 
even if we underestimate the correlation between these measures.

The most interesting results in Table 3 refer to the distribution of latent health for different education and age groups. The relevant parameters are reported in the rows 1 through 5 . These are difficult to interpret directly and we therefore show the implied distributions in Figures $3 \mathrm{a}, \mathrm{b}$, and c.

The first thing to notice is that there are very large deviations from normality. In particular, we observe that some of the distributions of latent 
health are bimodal. Indeed among those aged 50-54 with less than 12 years of education, we observe that the estimated distribution almost separates into two distinct normal distributions with very little overlap. 
Figure 4 then shows how mean latent health varies across age. The trends in health, as measured here, conform closely to the ones obtained from the objective health measures and presented in figure 2. The deterioration in health starts early and accelerates somewhat after age 70. Health differences across education are sizeable, but they are not as large compared to the age differences as those found using SRHS or the large muscle index only. 
Overall, this again underlines the importance of using distinct measures of health to estimate the dynamics of health.

\section{Estimating the Dynamic Model}

In this section we propose and implement a new estimation method to recover the parameters of the dynamic process, a method we will term simulated moment estimation of dynamic factors (SMDF). This method has the advantage that it can accommodate complex dynamic processes such as those that govern how individuals age. In this paper, we demonstrate the methodology using the simple dynamic process proposed in Section 2.

\subsection{A Simulated Method of Moments Approach to Es- timating the Dynamic Latent Health Process}

We propose a simulation based algorithm that minimizes the distance between moments obtained from simulated health measurement data and moments of the observed empirical distribution of measures. Let $\widetilde{Z}$ denote a simulated panel data-set containing measures of health for individuals at different ages. Based on $\widetilde{Z}$ we can compute simulated moments of the joint and marginal distribution of the measures. Denote these moments $\widetilde{M}(\theta)$, where the simulated moments depend on the parameter vector $\theta$ which is in turn composed of $\left\{\mu_{g}, \rho_{g}, \sigma_{v, g}^{2}, \alpha_{0, g}, \alpha_{1, g}\right\}_{g=0}^{G}$. We can construct the same moments from the observed data and will denote these moments 
$M$. Our estimator then chooses $\widehat{\theta}$ to minimize the distance $D(M-\widetilde{M}(\theta))=$ $(M-\widetilde{M}(\theta))^{\prime} W(M-\widetilde{M}(\theta))$ where $\mathrm{W}$ is an appropriately chosen weighting matrix.

At the core of the estimator is an algorithm that lets us construct $\widetilde{M}(\theta)$. Before we explain this algorithm, note that we can separately estimate the parameters of the mapping from the latent health variable into $Z_{a}$ (as shown in Section 4) and we can thus treat this mapping as known. Furthermore note that the static measurement model estimated at age 50 provides an estimate of the distribution of health for the age at which we are initializing the health model. To construct the simulated moments of measurements $\widetilde{M}(\theta)$, we use the following steps:

Step 1 Generate draws of initial health $\widetilde{h}_{0}$ for a large simulated sample of individuals by drawing from the estimated distribution $F_{0}($.$) of the$ latent health variable at the initial age $\mathrm{a}=0$.

Step 2 Use the dynamic model to draw $\widetilde{h}_{a+1} \mid \widetilde{h}_{a}$ for each individual. This generates a simulated panel of health histories.

Step 3 Use the implied survival probability $\Phi\left(\alpha_{0, g}+\alpha_{1, g} \widetilde{h}_{a}\right)$ to simulate the mortality process and generate a sample of survivors.

Step 4 For each age in the panel, use the estimates from the static measurement model to draw $\widetilde{z}_{a} \mid \widetilde{h}_{a}$ on the sample of survivors. In this manner, we have a panel of measurements $\left\{\widetilde{z}_{a}\right\}_{a=0}^{A}$ from which we can generate $\widetilde{M}(\theta)$ and for which we can generate the distance $D(M-\widetilde{M}(\theta))$. 
It should be clear that steps 3 and 4 can be implemented for very complex dynamic processes and that this proposed method therefore allows us to estimate dynamic models that are substantially more complex than our current AR-1 and mortality selection model.

\subsection{Implementing the Simulated Moments Algorithm}

The first requirement for implementing the above algorithm is to choose moments $M(\theta)$. By construction, the measurement model matches the crosssectional distribution of the health measures in a given age group. The moments that are available to estimate the dynamic models are therefore moments from the joint distribution of health measures in a and a+1. Because the marginal distribution of a is given by the static model parameters and we start our simulations at age a, we restrict ourselves to moments that include measures from period $a+1$.

1. For each continuous objective measure we match

(a) average change in the measure from a to $a+1$. (3 moments)

(b) variances in the objective measures in a+1 ( 3 moments).

(c) covariances in the measures between a and a+1 (3 moments).

2. For each categorical subjective measure, we match the entire intertemporal transition matrix. Both the large muscle index and the self-rated health index variable have 5 support points and there are therefore 25 
transition probabilities. The transition probabilities within each row of the transition matrix must sum to 1 implying 5 restrictions on the transition matrix. This means that each of the ordinal categorical variables contributes 20 moments for each of the age-transitions that we are considering $(2 * 20$ moments $)$.

3. To identify the survival process, we also match

(a) mean of the objective measures in a, conditional on dying before period $\mathrm{a}+1$ (3 moments).

(b) marginal distribution for each of the subjective measures, also conditional on dying before period a $+1(2 * 4$ moments $)$

(c) unconditional mortality rate (1 moment)

For each age group, we thus have a total of 61 moments that we use to fit the 5 parameters of $\theta\left(\mu_{g}, \rho_{g}, \sigma_{v, g}^{2}, \alpha_{0, g}, \alpha_{1, g}\right)$ for each age group. The estimated parameters will be asymptotically consistent regardless of the weighting matrix chosen, but in finite sample, the choice of the weighting matrix can lead to different estimates. We estimate our model using the conventional two step process. First, we use an identity weighting matrix to get a preliminary estimate of $\theta$. Then we use this estimate to construct an optimal weighting matrix (Hansen, 1982) and re-estimate. We have found little difference between the preliminary and optimally weighted estimates.

At present, we have not yet computed standard errors. We will use standard method of simulated moment techniques to obtain the standard errors 
of the dynamic parameters estimated conditional on a particular set of static parameters. We then propose using a parametric boot-strap on the parameters of the static measurement model to account for the estimation error in the static parameters.

\subsection{Estimates of the Dynamic Model}

Tables 5 and 6 contain the estimates of the dynamic model described above. To enable evaluation of the relevance of modeling mortality when estimating the health dynamics, we show estimates obtained when mortality is not included (Table 5) and estimates obtained for the full model including mortality selection (Table 6). Standard errors are not yet available. The parameter estimates from the model without mortality correction are reported in columns 2-6, while the last column summarizes the average decline in health implied by the parameters. This decline is calculated by taking $E\left[h_{a+1}\right]-E\left[h_{a}\right]=E\left[\mu+\rho h_{a}+\varepsilon_{a+1}\right]-E\left[h_{a}\right]=\mu+(\rho-1) E\left[h_{a}\right]$ and depends on the two parameters $(\mu, \rho)$ as well as the observed mean health for each age: $E\left[h_{a}\right]$. This decline is calculated without conditioning on survival - it shows what would happen to average health in populations that are not subject to mortality. ${ }^{23}$

\footnotetext{
${ }^{23}$ This is true even for Table 6 . In table 6 , the parameter estimates are obtained correcting for mortality, but the health declines shown in the last column represent mean health changes for a population that is not subject to mortality.
} 
Comparing the last columns in Tables 5 and 6 across education groups, we observe that declines in health are of similar magnitude for both education groups, except for very old individuals. For those older than 75, we observe larger declines in health among the educated relative to the less educated sample. This pattern is stronger for the estimates presented in Table 6 which 
correct for endogenous mortality based on latent health. Regardless whether we estimate our parameters correcting for mortality or not, we find that the predicted decline in health is stronger among the educated than the less educated at old age. Thus, the finding that health differences narrow across education groups during old age does not seem to be an artifact of selective mortality.

To understand the size of the health declines with age, it is useful to compare it to the within age-education standard deviation in the latent health variable. This standard deviation varies somewhat with age, but typically lies between 0.15 and $0.25 .^{24}$ The annual declines in average health are therefore comparable to about $1 / 3$ of a standard deviation of health within any given age group. We find these declines with age to be large (in other words, the dispersion in health within age across individuals is relatively small compared to the effect of aging).

We can also compare the volatility in health to the average declines in health that we observe. The estimates of $\sigma$ represent the standard deviation in the individual innovations to health. Depending on the age group, the estimates of $\sigma$ vary between close to 0 and about 0.1 - suggesting that individual health shocks are typically of about the same magnitude as the age gradient, even though a number of individuals experience relatively large declines. For instance, the standard deviation of 0.105 reported in Table 5

\footnotetext{
${ }^{24}$ Except at old age, when it increases to approximately 0.3 at ages $75-80$ and 0.4 for individuals aged 85 and more.
} 
for educated individuals aged 65-69 implies that on average $2.5 \%$ of the population experience a decline in health in each year that is about equivalent to the average decline in health experience during 7-8 years of aging (using the estimate of 0.028 reported for this group in the last column). Thus, while volatility is not enormous, we do predict quite large year-on-year declines in health for a non-trivial number of individuals.

When we consider the estimates of the mortality equation reported in Table 6, we find quite similar parameter values for the mortality equation estimated on both education groups and likewise across ages. It seems as if the mortality differences observed across education groups (see Cutler, et al 2008, 2009) are not driven by differences in mortality conditional on observed health, but are rather due to differences in distribution of the health variable between high school drop-outs and high school graduates.

Consider next the estimates for $\rho$, the parameter governing the persistence of health at the individual level. Our estimates for $\rho$ are typically very close to 1 for all ages, regardless for whether we account for mortality. ${ }^{25}$ If we take the estimates of $\rho$ that exceed 1 seriously, then we predict that individuals whose health falls short of the mean will on average experience even further relative deterioration of health. We find it plausible that individuals with bad health might experience continued relative deteriorations. After a health shock, individuals might enter a downward spiral that generates additional

\footnotetext{
${ }^{25}$ The estimates of $\rho$ from the mortality corrected model are somewhat smaller, but not across all age groups.
} 
declines in health, possibly resulting in death. However, the symmetry of the specification implies that for estimates $\rho>1$ we would likewise predict that healthy individuals will, on average, continue to improve in health relative to the mean in the population. This result is counterintuitive. One of our next steps in this project is to relax this assumption of symmetry and allow the level of persistence $(\rho)$ to vary based on whether individuals are above or below mean health.

Table 7 considers the fit of our model by presenting the observed (first entry in each cell) and predicted (second entry) correlations in our health measures across two years (the periodicity of the HRS). A number of patterns are noteworthy. First, within measures there is substantially more persistence than across health measures and our estimates generally do not pick up this feature of the data. However, we do a substantially better job in matching the cross-correlations between the subjective measures over time: for example, for high school graduates the predicted correlation between the large muscle index at $\mathrm{t}$ and the SHRS measure at $\mathrm{t}+2$ is 0.37 , whereas the observed is 0.36 . We have more difficulties reproducing the correlations of the objective measures across time periods and find some quite large differences. In particular, we find these differences to be large for the walking speed measure. We believe that these differences are due to the relatively small number of individuals for whom we have objective measures. This results in imprecisely estimated parameters of the measurement model and thus generates relatively noisy predicted correlations. This problem will be at least partially 
alleviated when we incorporate the 2008 data into our analysis. 


\subsection{What Have We Learned About the SES-Gradient? Some Speculative Results}

Our results are preliminary and we are working to refine both the static and the dynamic model. Nevertheless, we can speculate about what we have learned concerning the sources of the gradient. We have found that there are large differences in health across education at age 50 . We also found that the health process is close to a martingale $(\rho \approx 1)$ and that estimated parameters are quite similar across education groups. Finally, we found that the estimated parameters for the mortality function are quite similar across education, suggesting that conditional on health, mortality is similar across the two groups. Together these findings suggest that health differences are, on average, persistent across individuals and that average health differences are also quite persistent across groups, at least until about age 75 . Thus, our preliminary results suggest that health differences across these two education groups are driven predominantly by differences in the endowments of individuals entering the sample at age 50 , rather than due to differential health dynamics subsequent to age 50 .

\section{Conclusion}

In this paper, we propose a new simulation-based method of estimating rich aging processes over the life-cycle. Our approach splits the problem into two parts. The first is a static measurement stage that recovers a distribution 
of the latent health variable conditional on controls at each age. The static stage also delivers estimates of the mapping from latent health into observed health measurements at each stage. The second stage of the approach focuses on the dynamics. Using the estimates of the measurement model, we can generate simulated joint distributions of the manifest measurement variables implied by our model of dynamic health and our estimated measurement model. The estimation proceeds by choosing parameters of the dynamic model that minimize differences between cross-age moments in the simulated and observed distributions of measures.

To demonstrate how this method works, we estimate a simple model of health dynamics on panel data from the HRS. The estimates from the first (static) stage deliver distributions of the latent health variables that are highly non-normal. At all ages and education levels, we find evidence for skew, bimodality and thick tails (kurtosis $>3$ ). In the second stage, we estimate a simple dynamic model of health based on an AR-1 autoregressive structure that allows for mortality selection based on the latent health variable.

At the individual level, our results suggest that there is a substantial degree of health volatility, but that there is also a high degree of persistence in health. We find continuous declines in health over the life-cycle that are of about the same size for those with and without high school degrees. Only during old age are these differences in health narrowing. The decline in the SES-gradient in health observed after age 75 is however not due to mortality 
selection - indeed correcting for mortality selection strengthens the finding that the SES-gradient declines in old age.

Preliminary speculations suggest that differences in endowments at age 50 , rather than differences in the health dynamics after age 50 are generating the observed SES gradients. The question we face now is whether this preliminary speculative result will be robust to the refinements of the model we are planning next.

Regarding these refinements, we now find ourselves in the position to estimate more interesting dynamic models of health than the simple specification described above. Based on the results reported in this paper, we plan to enrich the analysis along two dimensions. First, we plan to allow for asymmetries in the persistence of health shocks - allowing for the possibility that individuals who find themselves with better than average health might regress back to the mean, while those with below average health might find themselves on a persistent downward trajectory. We also believe that the observed non-symmetries in the distribution of health (see Figures 3 a-c) warrant a further refinement of the dynamic model. In particular, we are hoping to integrate the data on health shocks in the HRS into our analysis. The HRS records when individuals experience serious health events such as heart attacks or diagnoses of cancer. Allowing the dynamics of health to vary conditional on experiencing such events may explain the bimodality and skew we observe in the static distributions of health. 


\section{Appendix: Identification}

The parameters that need to be identified are the dynamic parameters $\left(\mu_{a}, \rho_{a}, \sigma_{v, a}^{2}\right)$, the initial distribution of health $F\left(h_{0}\right)$, as well as the parameters from the measurement model $\left(\alpha_{a}, \Lambda_{a}, c_{j, a}^{k-1}\right)$. We will show that these parameters are identified up to a normalization on the intercept and factor loading for one of the continuous measurement equations, as well as the standard normalizations on variances and intercepts of categorical measurement equations. We assume that we have access to at least 2 continuous measurement variables and 3 additional continuous or categorical measurement variables.

First, we appeal to standard factor analytic arguments and assert that with 3 continuous and categorical measurement variables, we can identify the parameter vectors $\left(\alpha_{a}, \lambda_{a}\right)$ and the variances of $\epsilon_{a}$ up to a normalization of one intercept and one factor loading. We will impose these normalizations on the same measurement equation at all ages.

Because $F\left(h_{0}\right)$ was unrestricted, $F\left(h_{a}\right)$ is also unrestricted and we therefore will next show, using Kotlarski's Theorem, that $F\left(h_{a}\right)$ can be identified using two continuous measurements only. Use the first and second continuous measurement for this identification argument: $x_{1, a}$ and $x_{2, a}$. We have normalized the factor loading and intercept on the first and thus have

$$
\begin{aligned}
& x_{1, a}=h_{a}+\varepsilon_{1, a} \\
& x_{2, a}=\alpha_{2, a}+\lambda_{2, a} h_{a}+\varepsilon_{2, a}
\end{aligned}
$$


Since $\left(\alpha_{2}, \lambda_{2}\right)$ are identified, we can write:

$$
\begin{aligned}
x_{1, a} & =h_{a}+\varepsilon_{1, a} \\
\frac{x_{2, a}-\alpha_{2}, a}{\lambda_{2, a}} & =h_{a}+\frac{\varepsilon_{2, a}}{\lambda_{2, a}}
\end{aligned}
$$

and can treat the left hand side of both of these equations as observed. Kotlarski's Theorem implies that if $\left(h_{a}, \varepsilon_{1, a}, \frac{\varepsilon_{2, a}}{\lambda_{2, a}}\right)$ are jointly independent and $E\left[\varepsilon_{1, a}\right]=E\left[\frac{\varepsilon_{2, a}}{\lambda_{2, a}}\right]=0$, then the marginal distribution of $\mathrm{h}$ can be identified from the joint distribution of $\left(x_{1, a}, x_{2, a}\right)$. Therefore, $F\left(h_{a}\right)$ and the parameters of the measurement equations are identified.

We have yet to discuss the identification of the parameters of the dynamic equation (1) . For this purpose, we will restrict attention to two adjacent ages $(a, a+1)$. First, note that we can identify the parameters $\left\{\mu_{a}\right\}$ using the marginal distributions of health $\mathrm{h}_{a}$ directly:

$$
\begin{aligned}
\mu_{0} & =E\left[h_{0}\right] \\
E\left[h_{a+1}\right] & =\mu_{a}+\rho_{a} E\left[h_{a}\right]
\end{aligned}
$$

Now, from equation (1) we get:

$$
\begin{aligned}
E\left[h_{a+1} \mid Z_{a}\right] & =\mu_{a}+\rho_{a} E\left[h_{a} \mid Z_{a}\right]+E\left[\varepsilon_{a+1} \mid Z_{a}\right] \\
& \Longrightarrow \rho_{a}=\frac{E\left[h_{a+1} \mid Z_{a}\right]-\mu_{a}}{E\left[h_{a} \mid Z_{a}\right]}
\end{aligned}
$$


$E\left[h_{a} \mid Z_{a}\right]$ can be directly obtained using the parameter estimates from the measurement model. However, we do not have direct estimates of $E\left[h_{a+1} \mid Z_{a}\right] .{ }^{26}$ However, we have the following:

$$
\begin{aligned}
E\left[Z_{a+1} \mid Z_{a}\right] & =E\left[\alpha_{a+1}+\Lambda_{a+1} h_{a+1}+\varepsilon_{a+1} \mid Z_{a}\right] \\
& =\alpha_{a+1}+\Lambda_{a+1} E\left[h_{a+1} \mid Z_{a}\right] \\
& \Leftrightarrow E\left[h_{a+1} \mid Z_{a}\right]=\left(\Lambda_{a+1}^{\prime} \Lambda_{a+1}\right)^{-1} \Lambda_{a+1}^{\prime}\left(E\left[Z_{a+1} \mid Z_{a}\right]-\alpha_{a+1}\right)
\end{aligned}
$$

where the right hand side can be obtained using the estimated factor loading matrices and the data. Therefore $\rho_{a}$ is identified.

To identify $\sigma_{a}^{2}$, consider the following expression:

$$
\begin{aligned}
\operatorname{Var}\left(h_{a+1} \mid Z_{a}\right) & =V\left(\mu_{a}+\rho_{a} h_{a}+\varepsilon_{a+1} \mid Z_{a}\right) \\
& =\rho_{a}^{2} V\left(h_{a} \mid Z_{a}\right)+V\left(\varepsilon_{a+1} \mid Z_{a}\right) \\
& \Longrightarrow \sigma_{a}^{2}=\operatorname{Var}\left(h_{a+1} \mid Z_{a}\right)-\rho_{a}^{2} V\left(h_{a} \mid Z_{a}\right)
\end{aligned}
$$

Again, we obtain $V\left(h_{a} \mid Z_{a}\right)$ directly from the measurement model and we need to concern ourselves only with finding $\operatorname{Var}\left(h_{a+1} \mid Z_{a}\right)$. For this purpose, we again use the joint distribution of the measurement equations.

$$
\begin{aligned}
V\left(Z_{a+1} \mid Z_{a}\right) & =V\left(\alpha_{a+1}+\Lambda_{a+1} h_{a+1}+\varepsilon_{a+1} \mid Z_{a}\right)=\Lambda_{a+1} V\left(h_{a+1} \mid Z_{a}\right) \Lambda_{a+1}^{\prime}+V\left(\varepsilon_{a+1} \mid Z_{a}\right) \\
& =\Lambda_{a+1} V\left(h_{a+1} \mid Z_{a}\right) \Lambda_{a+1}^{\prime}+V\left(\varepsilon_{a+1}\right)
\end{aligned}
$$

\footnotetext{
${ }^{26}$ Note that $E\left[h_{a+1} \mid Z_{a}\right] \neq E\left[E\left[h_{a+1} \mid Z_{a+1}\right] \mid Z_{a}\right]$.
} 


$$
\Leftrightarrow V\left(h_{a+1} \mid Z_{a}\right)=\left(\Lambda_{a+1}^{\prime} \Lambda_{a+1}\right)^{-1} \Lambda_{a+1}^{\prime}\left(V\left(Z_{a+1} \mid Z_{a}\right)-V\left(\varepsilon_{a+1}\right)\right) \Lambda_{a+1}\left(\Lambda_{a+1}^{\prime} \Lambda_{a+1}\right)^{-1}
$$

where again the right-hand side is observed or estimable from the static model.

\section{References}

Adams, P., M.D. Hurd, D. McFadden, A. Merrill, and T. Ribeiro (2003), "Healthy, Wealthy, and wise? Tests for direct causal paths between health and economic status." Journal of Econometrics 112: 3-56. Bollen, K.A. (1989). Structural Equations with Latent Variables. Wiley, New York.

Cutler, D., F. Lange, E. Meara, S. Richards, and C. Ruhm (2009) Can Educational Gradients in Smoking and Obesity Explain Educational Gradients in Mortality?, available at: http://www.aeaweb.org/annual_mtg_papers/2009/ retrieve.php?pdfid $=506$

Du, et al. (2007) Racial Disparities and Socioeconomic Status in Association With Survival in a Large Population-based Cohort of Elderly Patients With Colon Cancer, Aug. 1, 2007, Cancer, Vol. 110, No. 3.

Gornick ME, Eggers PW, Riley GF (2004). Associations of race, education, and patterns of preventive service use with stage of cancer at time of diagnosis. Health Serv Res. 2004;39:1403-1427.

Halliday, Timothy (2008), "Heterogeneity, State Dependence and Health" Econometrics Journal, Vol. 11, Issue 3 (Nov. 2008), pp. 499-516. 
Halliday, Timothy (2009), "Health Inequality over the Lifecycle", IZA Discussion Paper, No. 4369.

Hayashi, K., P. Bentler, and K-H Yuan (2008), "Structural Equation Modeling", Handbook of Statistics, Vol. 27. Elsevier 2008.

Heiss, F., Börsch-Supan, M. Hurd, and D. Wise (2009), "Pathways to Disability: Predicting Health Trajectories", in Health at Older Ages, eds. David Cutler and David A. Wise, NBER Conference Report, University of Chicago Press.

E.M. Kitagawa and P.M. Hauser, Differential Mortality in the United States: A Study in Socioeconomic Epidemiology (Cambridge, MA: Harvard University Press, 1973).

Lleras-Muney, Adriana. The Relationship Between Education and Adult Mortality in the United States. Review of Economic Studies 2005;72:189-221.

Meara, E., S. Richards, and D. Cutler (2008) "The Gap Gets Bigger: Changes in Mortality and Life Expectancy by Education, 1981-2000", Health Affairs, 27, no. 2 (2008): 350-360

Meet, J., D. L. Miller, and H. S. Rosen, "Exploring the health-wealth nexus", Journal of Health Economics 22 (2003) 713-730.

Meijer, Erik, Arie Kaptyn, and Tatiana Andreyeva (2008), "Health Indezes and Retirement in Modeling in International Comparisons", Rand Working Paper WR-614.

Roberts, Stephanie and James S. House (1996) "SES Differentials in Health by Age and Alternative Indicators of SES" Journal of Aging and 
Health 1996, 8, 359.

Schoeni R.F., Freedman, V.A., and L.G. Martin (2009) "Socioeconomic and Demographic Disparities in Trends in Old-Age Disability", in Health at Older Ages, eds. David Cutler and David A. Wise, NBER Conference Report, University of Chicago Press.

Schoeni et al. (2005), "Persistent and Growing Socioeconomic Disparities in Disability Among the Elderly: 1982-2002," American Journal of Public Health, 2005; 95, no. 11: 2065-2070;

Steenland, K., Henley, J. and M. Thun (2002), All-Cause and Causespecific Death Rates by Educational Status for Two Million People in Two American Cancer Society Cohorts, 1959-1996, American Journal of Epidemiology 2002, Vol. 156, No.1.

James P. Smith (2007), Diabetes and the Rise of the SES Health Gradient, February 2007, NBER Working Paper No. W12905 available at: http://www.nber.org/papers/w12905 


\section{RECENT WORKING PAPERS FROM THE}

\section{CENTER FOR RETIREMENT RESEARCH AT BOSTON COLLEGE}

Getting to the Top of Mind: How Reminders Increase Saving

Dean Karlan, Margaret McConnell, Sendhil Mullainathan, and Jonathan Zinman, April 2010

Social Security, Benefit Claiming and Labor Force Participation: A Quantitative General Equilibrium Approach

Selahattin Imrohoroğlu and Sagiri Kitao, March 2010

How Much Is Enough? The Distribution of Lifetime Health Care Costs

Anthony Webb and Natalia Zhivan, February 2010

Impact of Immigration on the Distribution of American Well-Being Gary Burtless, December 2009

Actual and Anticipated Inheritance Receipts

Norma B. Coe and Anthony Webb, December 2009

Will Automatic Enrollment Reduce Employer Contributions to 401(k) Plans

Mauricio Soto and Barbara A. Butrica, December 2009

How Seniors Change Their Asset Holdings During Retirement

Karen Smith, Mauricio Soto, and Rudolph G. Penner, December 2009

Retirement Security and the Stock Market Crash: What Are the Possible Outcomes?

Barbara A. Butrica, Karen E. Smith, and Eric J. Toder, November 2009

Fees and Trading Costs of Equity Mutual Funds in 401(k) Plans and Potential Savings from ETFs and Commingled Trusts

Richard W. Kopcke, Francis Vitagliano, and Zhenya S. Karamcheva, November 2009

Work Ability and the Social Insurance Safety Net in the Years Prior to Retirement Richard W. Johnson, Melissa M. Favreault, and Corina Mommaerts, November 2009

Dutch Pension Funds in Underfunding: Solving Generational Dilemmas

Niels Kortleve and Eduard Ponds, November 2009

The Wealth of Older Americans and the Sub-Prime Debacle

Barry Bosworth and Rosanna Smart, November 2009

All working papers are available on the Center for Retirement Research website

(http://crr.bc.edu) and can be requested by e-mail (crr@bc.edu) or phone (617-552-1762). 